\title{
Lipoplex-Loaded Microbubbles for Gene Delivery: A Trojan Horse Controlled by Ultrasound**
}

\author{
By Ine Lentacker, Stefaan C. De Smedt, *Joseph Demeester, Veerle Van Marck, Marc Bracke, \\ and Niek N. Sanders
}

Cationic poly(ethylene glycol)ylated (PEGylated) liposomes are one of the most important gene transfer reagents in non-viral gene therapy. However, the low transfection efficiencies of highly PEGylated lipoplexes currently hamper their clinical use. Recently, ultrasound has been used in combination with microbubbles to enhance the uptake of genes in different cell types. However, the gene transfer efficiency still remains low in these experiments. To overcome the limitations of both techniques, we present the attachment of PEGylated lipoplexes to microbubbles via biotin-avidin-biotin linkages. Exposure of these lipoplex-loaded microbubbles to ultrasound results in the release of unaltered lipoplexes. Furthermore, these lipoplex-loaded microbubbles exhibit much higher transfection efficiencies than "free" PEGylated lipoplexes or naked plasmid DNA (pDNA) when combined with microbubbles and ultrasound. Interestingly, the lipoplex-loaded microbubbles only transfect cells when exposed to ultrasound, which is promising for space- and time-controlled gene transfer. Finally, this novel Trojan-horse-like concept can also be exploited to achieve the ultrasound-triggered release of nanoparticles containing other therapeutic agents such as anticancer drugs.

\section{Introduction}

The use of nucleic acids to replace defective genes or silence aberrant ones is an attractive strategy for the treatment of genetic disorders. However, the development of efficient and safe materials that can deliver therapeutic nucleic acids to the target cells of a patient is an enormous challenge. Both viral and non-viral nucleic acid delivery systems, such as cationic liposomes and cationic polymers, which electrostatically bind to negatively charged nucleic acids, are currently being investigated ${ }^{[1]}$ Unfortunately, the former system is plagued by safety issues, ${ }^{[2]}$ whereas the latter systems are vulnerable to non-specific interactions with blood compounds due to their positive surface charge, resulting in the formation of life-threatening aggregates and suffering from rapid clearance by the mononuclear phagocyte system. ${ }^{[3,4]}$ These unwanted interactions have been prevented by coating the non-viral gene delivery systems

[*] Prof. S. C. De Smedt, I. Lentacker, Prof. J. Demeester, Dr. N. N. Sanders

Labaratory of General Biochemistry and Physical Pharmacy

Faculty of Pharmaceutical Sciences

Harelbekestraat 72, 9000 Ghent (Belgium)

E-mail: stefaan.desmedt@ugent.be

Dr. V. Van Marck, Prof. M. Bracke

Laboratory of Experimental Cancerology

Department of Radiotherapy and Nuclear Medicine

Ghent University Hospital

De Pintelaan 185, 9000 Ghent (Belgium)

[**] Katharzyna Smigielska is thanked for practical assistance. I.L. is a doctoral fellow supported by the Fund for Scientific Research-Flanders (Belgium). N.N.S. is a Postdoctoral Fellow supported by the Fund for Scientific Research-Flanders (Belgium). Financial support from FWO and BOF-Ghent are acknowledged. with non-fouling polymers like poly(ethylene glycol) (PEG).$^{[5]}$ However, 'PEGylation' drastically reduces the transfection efficacy of non-viral gene delivery systems, which has been attributed to reduced cellular uptake and/or limited endosomal escape ${ }^{[6,7]}$ To overcome these limitations, the use of ultrasound energy in combination with gas-filled microbubbles has recently been proposed. ${ }^{[8-14]}$ Ultrasound may mediate the intracellular delivery of nucleic acids by the formation of small (100 to a few hundreds of nanometers large) transient pores in cell membranes. ${ }^{[15,16]}$ These cell perforations are caused by shockwaves and microjets that are generated by the ultrasound-induced implosion of microbubbles in the vicinity of the cell membranes. The lifetimes of the cell membrane perforations have been reported to be very short, in the millisecond to seconds range. ${ }^{[15-17]}$ Therefore, we believe that the binding of nanoparticles to microbubbles will allow the particles to be present at the site of cell membrane perforation, which may enhance the number of nanoparticles that can enter the cell. In this study, we have attempted to load PEGylated lipoplexes onto microbubbles to overcome their low transfection efficiency. Furthermore, the coupling of liposomes to microbubbles could be an interesting approach for the ultrasound-induced release of different types of therapeutic molecules.

\section{Results and Discussion}

\subsection{Preparation and Characterization of Lipoplex-Loaded Microbubbles}

As schematically illustrated in Figure 1A, PEGylated lipoplexes, i.e., complexes of DNA with cationic PEGylated liposomes (Fig. 1B), have been attached to microbubbles via 


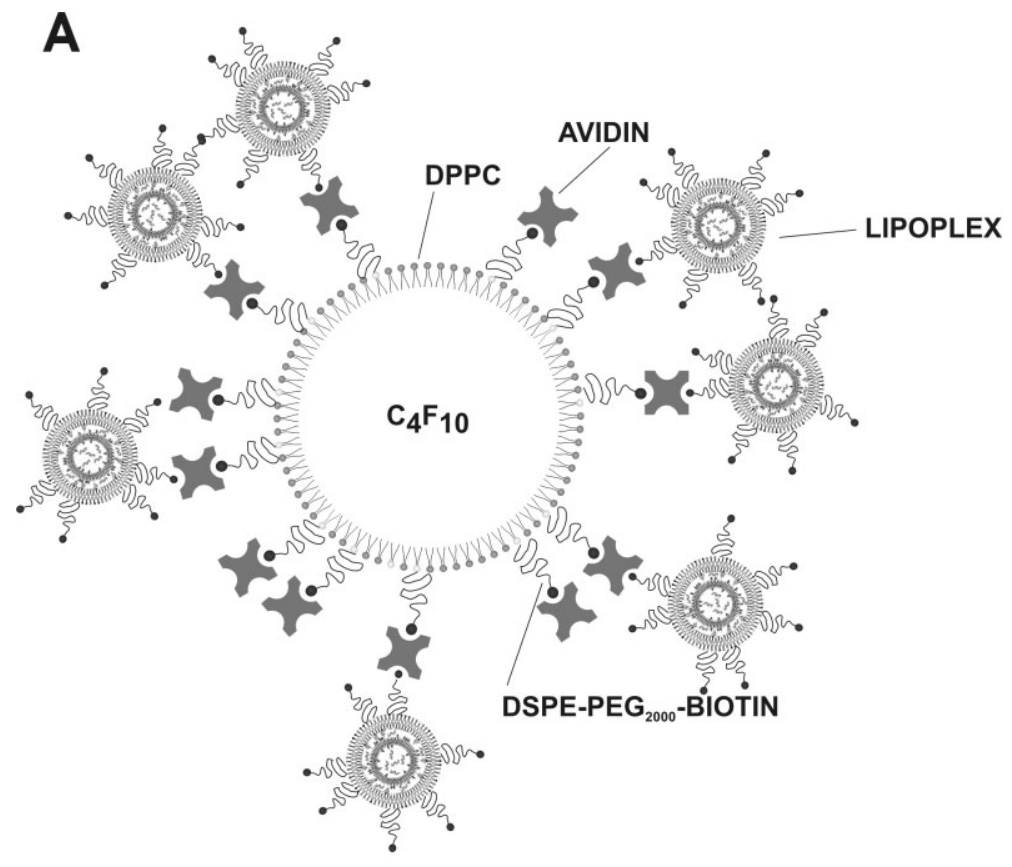

B

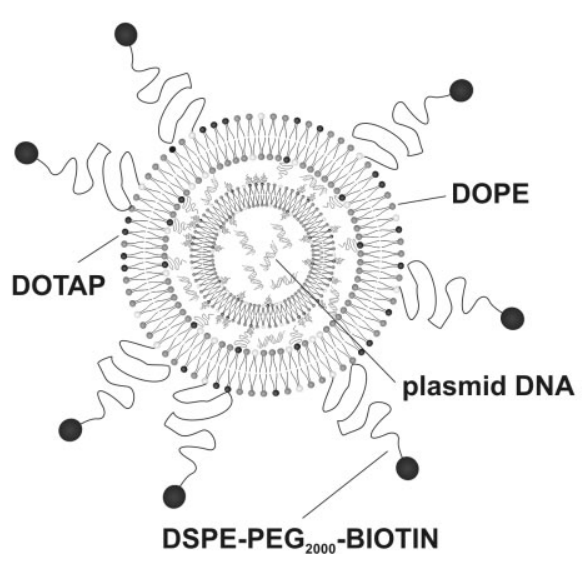

Figure 1. A) Schematic depiction of a lipoplex-loaded microbubble. The white disk surrounded by the lipids, dipalmitoylphosphatidylcholine (DPPC) and 1,2-distearoylsn-glycero-3-phosphoethanolamine- $N$-[biotinyl-PEG-2000] (DSPE-PEG-biotin), represents an avidinylated lipid microbubble with a perfluorobutane $\left(\mathrm{C}_{4} \mathrm{~F}_{10}\right)$ gas core. Lipoplexes with increasing amounts of DSPE-PEG-biotin are attached to these avidinylated microbubbles via biotin-avidin-biotin bridges. B) Detailed illustration of a single biotinylated lipoplex. DOTAP: $N$-(1-(2,3-dioleoyloxy) propyl)-N,N,N-trimethylammonium chloride; DOPE: dioleoyl phosphatidyl ethanolamine.

biotin-avidin-biotin bridges. To prepare these lipoplex-loaded microbubbles, we have used lipid-based microbubbles that contain 1,2-distearoyl-sn-glycero-3-phosphoethanolamine- $N$-[biotinyl-PEG-2000] (DSPE-PEG-biotin) in their lipid shell. To evaluate whether the biotin molecules are present on the outer surface of the microbubbles, we have incubated them with Cy5-labeled avidin. After removing the free Cy5-avidin, confocal laser scanning microscopy (CLSM) clearly reveals the presence of red fluorescence around the microbubbles, indicating the formation of a biotin-avidin linkage (Fig. 2). This confirms that the DSPE-PEG-biotin molecules in the shell of the microbubbles are oriented with their hydrophobic tails pointing to the perfluorobutane gas core and with their hydrophilic head groups in the aqueous medium, as previously suggested by Unger et al. ${ }^{[18]}$ To determine whether the biotinylated microbubbles have an optimal size distribution for cavitation, we have studied their size distribution by laser diffraction (Fig. 3, solid circles). As shown in Figure 3, the microbubbles are between 0.5 and $10 \mu \mathrm{m}$ in size, which is indeed optimal for cavitation upon exposure to clinically relevant ultrasound irradiation. ${ }^{[18]}$ To enable the binding of the PEGylated lipoplexes to the biotin-containing microbubbles, we have first incubated the microbubbles with an excess of avidin. After removing the unbound avidin, PEGylated lipoplexes containing increasing amounts of DSPE-PEG-biotin $(2,5$, and $15 \mathrm{~mol} \%)$ are added to the microbubbles. Figure 4 demonstrates the binding of fluorescently-labeled PEGylated lipoplexes to the microbubbles. We observe that all the microbubbles have attached lipoplexes on their surface independent of the percentage of DSPE-PEG-biotin in the lipoplexes, indicating that the number of lipoplexes greatly exceeds the number of microbubbles. The amount of DSPE-PEG-biotin in the PEGylated lipoplexes clearly effects on the number of lipoplexes bound per microbubble. Lipoplexes containing 2 mol \% DSPE-PEG-biotin do not completely cover the surface of the microbubbles, likely because of the limited degree of biotinylation of the lipoplexes. The surfaces of most microbubbles are fully covered with PEGylated lipoplexes when 5 mol \% DSPE-PEGbiotin is incorporated in the lipoplexes, whereas all the microbubbles are completely covered with PEGylated lipoplexes when lipoplexes containing $15 \mathrm{~mol} \%$ DSPE-PEG-biotin are used. The inset to Figure $4 \mathrm{~B}$ shows a high-magnification image of a single lipoplex-loaded microbubble. To determine the effect of lipoplex binding on the size of the microbubbles, we have also measured the size distribution of the microbubbles after incubation with the biotinylated lipoplexes (Fig. 3, solid squares). Compared to the unloaded microbubbles, the lipoplexloaded microbubbles are slightly smaller, which is probably due to experimental variations such as localization of the sonication probe and removal of the subnatant during successive washing steps. To confirm that the lipoplexes are specifically bound to the microbubbles through avidin-biotin interactions, we have tested the binding of PEGylated lipoplexes lacking biotin. The lipoplexes used in this control experiment contain $5 \mathrm{~mol} \%$ DSPE-PEG instead of DSPE-PEG-biotin. The moderate binding of these non-biotinylated lipoplexes to the microbubbles is observed (data not shown). Since avidin is a glycosylated protein, non-specific interactions with different types of molecules and particulate matter can be expected. 


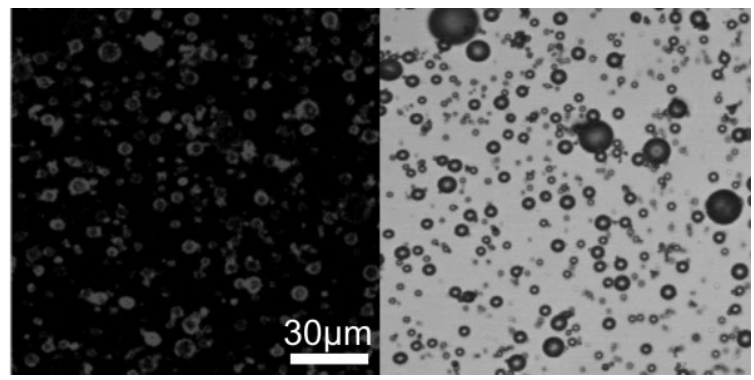

Figure 2. CLSM (left) and transmission (right) image of biotinylated microbubbles after incubation with Cy5-labeled streptavidin.

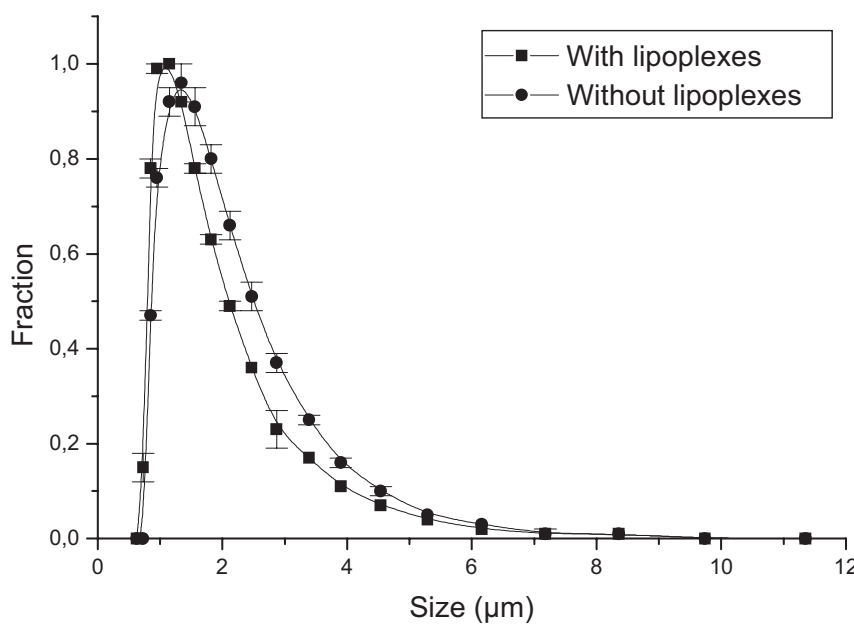

Figure 3. Size distribution of DPPC/DSPE-PEG-biotin microbubbles before and after the addition of $15 \mathrm{~mol} \%$ PEGylated lipoplexes, as measured by laser diffraction. The $y$-axis shows the abundance of a certain class of microbubbles normalized to the most abundant fraction of microbubbles $(y=1)$. The data represent the mean of three independent measurements.

\subsection{Ultrasound-Induced Release of PEGylated Lipoplexes from Microbubbles}

As mentioned in Section 1, the exposure of microbubbles to ultrasound causes the implosion and hence destruction of the microbubbles. Consequently, nanoparticles that are attached to the lipid-based microbubbles can be released from the microbubbles by ultrasound. Lum et al. ${ }^{[19]}$ have succeeded in attaching latex beads to lipid microbubbles, and have demonstrated the release of these beads under ultrasonic treatment. However, in contrast to inert beads, lipoplexes, which arise from the self-assembly of cationic liposomes and DNA, may undergo physicochemical alterations (which can possibly reduce their biological performance) during ultrasound-triggered release from the microbubbles. Therefore, some physicochemical properties of the lipoplexes have been measured before attachment to the microbubbles and after ultrasound-triggered release from the microbubbles. The size of the free lipoplexes has been measured using dynamic light scattering (DLS). The lipoplexes decrease in size (from ca. 325 to $125 \mathrm{~nm}$ ) with increasing degree of PEGylation. No significant difference in size is
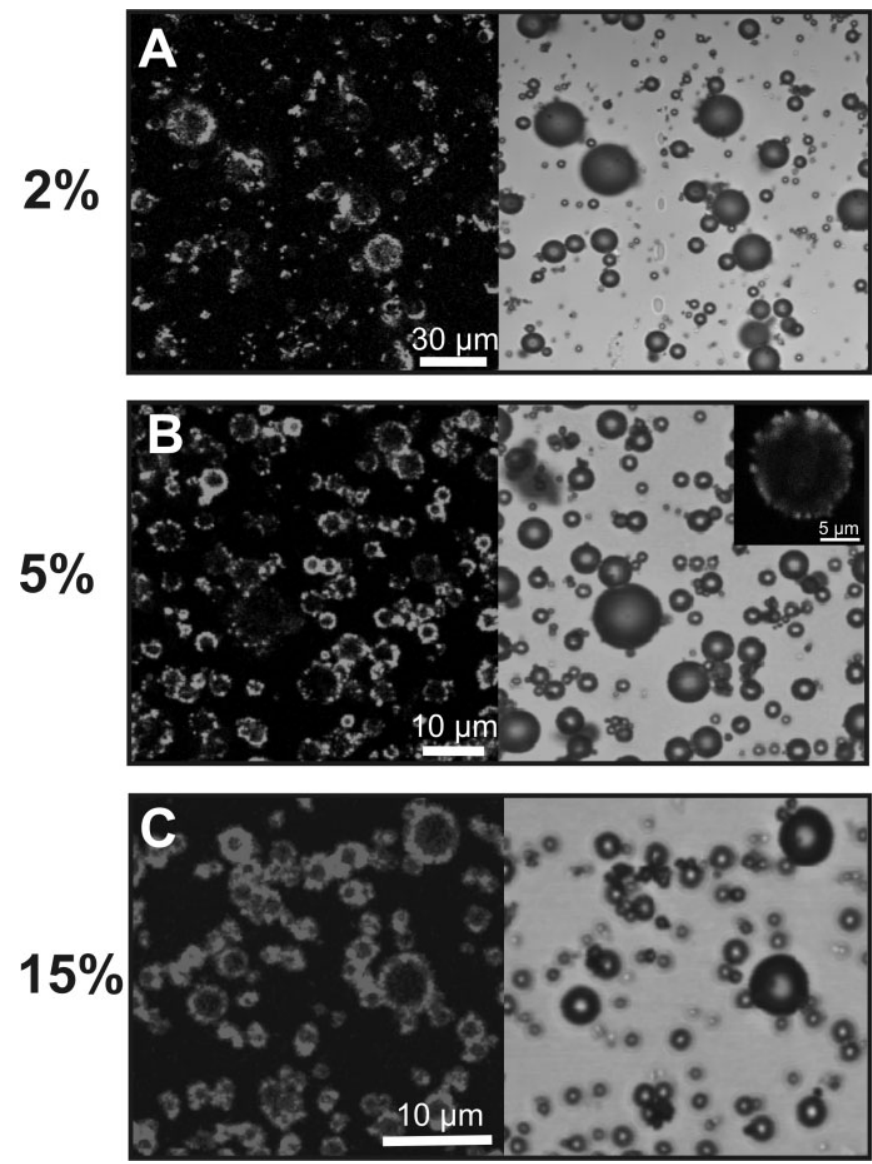

Figure 4. CLSM and corresponding transmission images of avidinylated microbubbles incubated with fluorescently labeled (green) PEGylated lipoplexes. The PEGylated lipoplexes contain A) $2 \mathrm{~mol} \%$, B) $5 \mathrm{~mol} \%$, and C) $15 \mathrm{~mol} \%$ DSPE-PEG-biotin. The inset to (B) shows a microbubble with single lipoplexes on its surface.

observed after the ultrasound-induced release of the lipoplexes from the microbubbles, except that the lipoplexes prepared with $15 \mathrm{~mol} \%$ DPPC-PEG-biotin become slightly larger (Fig. 5). The zeta potential before attachment to the microbubbles is around $25 \mathrm{mV}$ for lipoplexes containing $2 \mathrm{~mol} \%$ DSPE-PEG-biotin, around $22 \mathrm{mV}$ for lipoplexes with $5 \mathrm{~mol} \%$ DSPE-PEG-biotin, and ca. $14 \mathrm{mV}$ for lipoplexes containing $15 \mathrm{~mol} \%$ DSPE-PEG-biotin. The zeta potential of the lipoplexes is not significantly altered after release from the microbubbles by ultrasound treatment (Fig. 6). Apart from the retention of the size and zeta potential of the lipoplexes, it is also important that they do not dissociate upon exposure to ultrasound. Therefore, gel electrophoresis has been used to evaluate whether the ultrasound-assisted release of lipoplexes from the microbubbles leads to the release of plasmid DNA (pDNA). Free pDNA is not detected before the attachment of the lipoplexes or after the ultrasound-induced release of the lipoplexes from the microbubbles (Fig. 7). This means that the ultrasound-mediated implosion of the microbubbles and the induced microjets do not influence the complexation properties of the cationic liposomes. 
Before sonication

After sonication with microbubbles

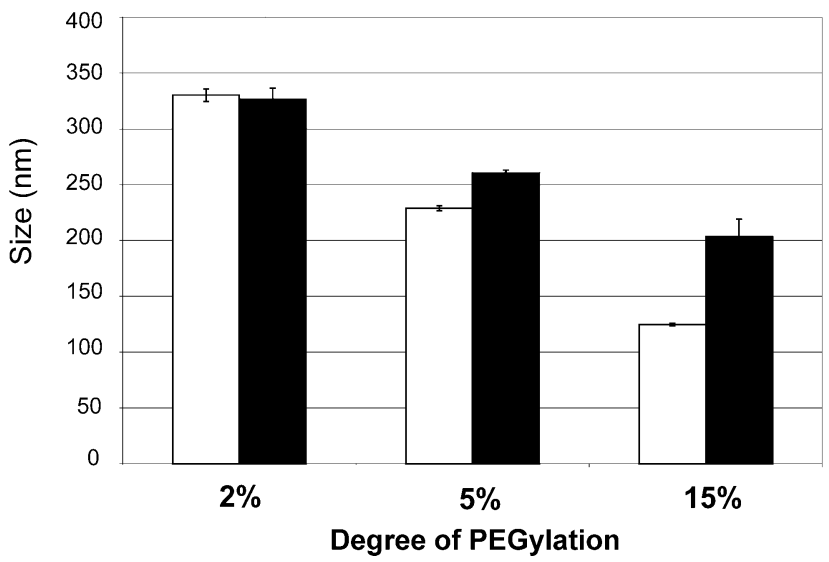

Figure 5. Sizes of PEGylated lipoplexes containing 2, 5, and $15 \mathrm{~mol} \%$ DSPE-PEG-biotin before attachment to the microbubbles (white bars) and after ultrasound-mediated release from the microbubbles (black bars). The data represent the mean of three independent measurements and the error bars indicate the standard deviation.

Before sonication

After sonication with microbubbles

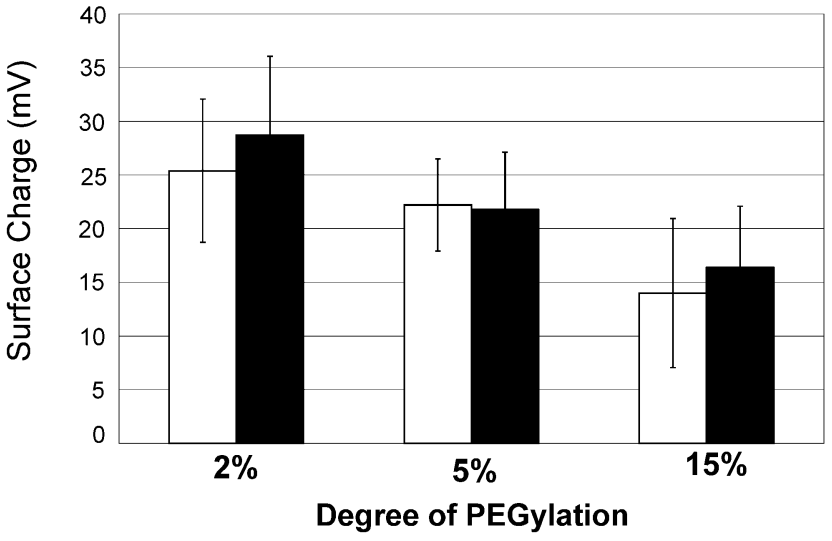

Figure 6. Zeta potential of PEGylated lipoplexes containing 2, 5, and $15 \mathrm{~mol} \%$ DSPE-PEG-biotin before attachment to the microbubbles (white bars) and after ultrasound-mediated release from the microbubbles (black bars). The data represent the mean of three independent measurements and the error bars indicate the standard deviation.

\subsection{Gene Transfer Efficiency of Lipoplex-Bearing Microbubbles}

The gene transfer efficacies of PEGylated lipoplexes, a (physical) mixture of PEGylated lipoplexes and microbubbles, and the lipoplex-loaded microbubbles have been evaluated. As shown in Figure 8 (white bars, part B), the higher the degree of PEGylation of the lipoplexes, the lower their transfection capacity. Lipoplexes with a degree of PEGylation of $5 \mathrm{~mol} \%$ are only slightly better than naked DNA (part A in Fig. 8),

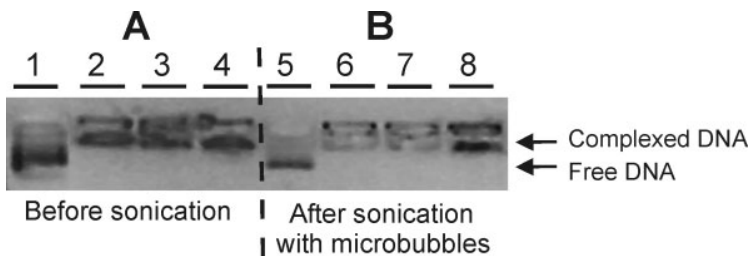

Figure 7. Gel electrophoresis of PEGylated lipoplexes containing 2, 5, and 15 mol\% DSPE-PEG-biotin: A) before attachment to the microbubbles and $B)$ after ultrasound-mediated release from the microbubbles. A) Lane 1: free pDNA; Lanes 2-4: PEGylated lipoplexes prepared with in creasing amounts of DSPE-PEG-biotin, 2, 5, and $15 \mathrm{~mol} \%$, respectively. B) Lane 5: free pDNA; Lanes 6-8: PEGylated lipoplexes prepared with increasing amounts of DSPE-PEG-biotin, 2, 5, and $15 \mathrm{~mol} \%$, respectively.

whereas lipoplexes with a degree of PEGylation of $15 \mathrm{~mol} \%$ show almost no transfection. The failure of highly PEGylated lipoplexes to transfect cells is in agreement with results of other research groups, and has been ascribed to both a reduced cellular uptake ${ }^{[20]}$ and an inhibition of the endosomal release of DNA into the cytoplasm by the PEG lipids in the lipoplexes. ${ }^{[21-25]}$ Figure 8 (grey bars, part B) also shows data for the transfection of cells when they are exposed to a (physical) mixture of PEGylated lipoplexes using microbubbles and ultrasound. Since the ultrasound energy itself does not alter the physicochemical properties of the PEGylated lipoplexes (see discussion above), it is reasonable to expect that the transfection of the cells should at least be similar to that observed when only free PEGylated lipoplexes are used (white bars, part B of Fig. 8). However, we hypothesize that the strong decrease in gene transfer observed for the $2 \mathrm{~mol} \%$ PEGylated lipoplexes arises from an ultrasound-induced blockage of endocytosis, which is line with previous observations by Schlicher et al. ${ }^{[16]}$ They have shown that ultrasound treatment in the presence of microbubbles removes patches of the plasma membrane; these are subsequently resealed by lipid vesicles transported from the inside of the cell to the plasma membrane (exocytosis). The endocytosis of the cells, which is the major mechanism for the uptake of the lipoplexes, may be significantly altered upon exposure to ultrasound irradiation. Such repair mechanisms of the cell membrane may prevent the endocytotic uptake of the lipoplexes. ${ }^{[26]}$ Figure 8 (grey bars, part B) shows that the presence of microbubbles and the application of ultrasound does not really reduce the transfection properties of 5 and $15 \mathrm{~mol} \%$ PEGylated lipoplexes. Since the 5 and $15 \mathrm{~mol} \%$ PEGylated lipoplexes are much less endocytozed by the cells, it is reasonable to expect that the (negative) influence of ultrasound and microbubbles will be much less pronounced. Importantly, Figure 8 (black bars, part B) clearly shows that the attachment of the lipoplexes to the microbubbles tremendously increases the transfection efficiency of the $5 \mathrm{~mol} \%$, and especially the $15 \mathrm{~mol} \%$, PEGylated lipoplexes. As noted above, free PEGylated lipoplexes encounter difficulties in entering the cells and/ or in escaping from endosomes, especially when they are highly PEGylated. ${ }^{[20,22-25]}$ We hypothesize that most of the PEGylated lipoplexes released from the microbubbles do not enter the cells by endocytosis, and consequently do not have to escape 
Naked DNA

Naked DNA with microbubbles (with ultrasound)

Free lipoplexes without microbubbles (without ultrasound)

Free lipoplexes with microbubbles (with ultrasound)

Lipoplex loaded microbubbles (with ultrasound)

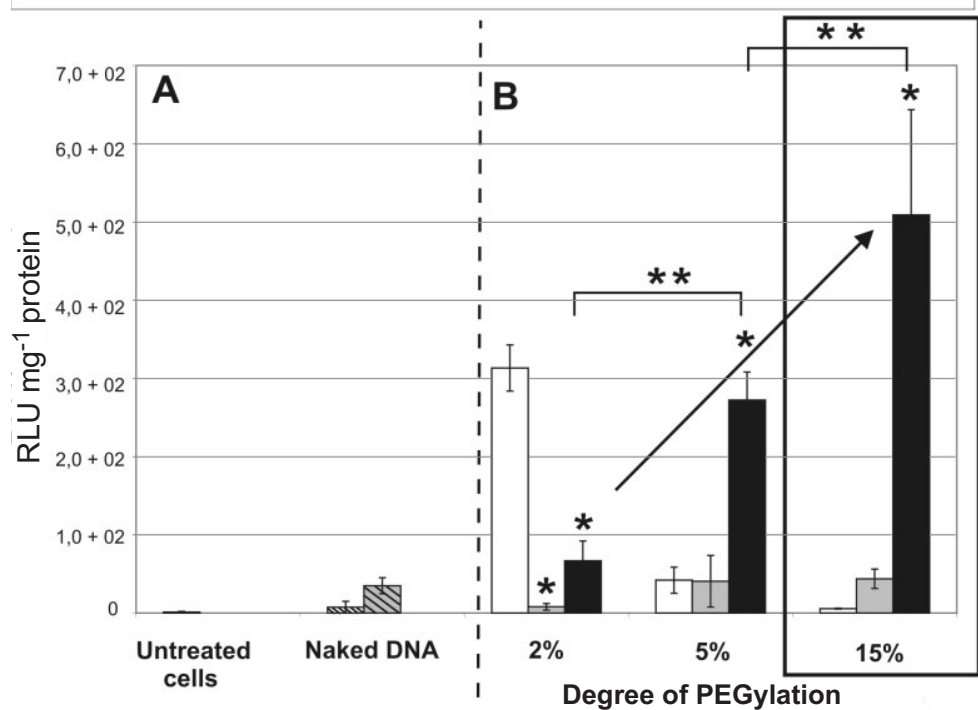

Figure 8. A) The transfection efficiency of naked DNA in the absence and presence of microbubbles and ultrasound, and the background luciferase signal in untreated cells. B) The transfection efficiency of free PEGylated lipoplexes in the absence and presence of microbubbles and ultrasound, and that of lipoplex-loaded microbubbles in the presence of ultrasound. The transfection results, i.e., the extent of luciferase expression, are expressed as RLU mg ${ }^{-1}$ (RLU: relative light units) protein. * $p<0.05$, compared to lipoplexes or naked DNA without microbubbles and ultrasound. $* * p<0.05$.

explained as follows: firstly, more PEGylated lipoplexes are attached to the microbubbles when the lipoplexes contain more DSPE-PEG-biotin, which results in an increased concentration of PEGylated lipoplexes at the cell membrane perforations. Secondly, we note that the higher the degree of PEGylation, the smaller the size of the lipoplexes released from the microbubbles, which increases the chances of more PEGylated lipoplexes passing through the cell perforations. Finally, when no ultrasound is applied, the cell transfection by lipoplex-loaded microbubbles is negligible, and even lower than the transfection by free PEGylated lipoplexes (data not shown). This is again reasonable since without ultrasound the lipoplexes remain attached to the micrometer-sized bubbles that are too large to enter the cells. Interestingly, this may enable the microbubbles to transfect only those cells that are exposed to ultrasound energy, which may be promising for targeted in vivo gene delivery.

\section{Conclusions}

We have designed a novel lipid microbubble to which PEGylated lipoplexes are attached via biotinavidin-biotin linkages. Upon exposure to ultrasound, unaltered lipoplexes are released from these lipoplex-loaded microbubbles. This is in contrast to previously developed layer-by-layer coated microbubbles, which lead to the release of undefined DNAcontaining clusters that are too large to pass through the small cell perforations. ${ }^{[29]}$ The lipoplex-loaded microbubbles have a much higher gene transfer ca-

from the endosomes. To verify this hypothesis, we have studied the cellular uptake of the $15 \mathrm{~mol} \%$ PEGylated lipoplexes after exposure to ultrasound. Figure 9 shows a massive internalization of the fluorescently labeled $15 \mathrm{~mol} \%$ PEGylated lipoplexes immediately after exposure of the melanoma cells to lipoplex-loaded microbubbles and ultrasound. As mentioned above, several groups have reported the formation of transient cell membrane perforations upon the implosion of microbubbles at or near cell membranes. ${ }^{[15,16,27,28]}$ Consequently, it is reasonable to expect that the PEGylated lipoplexes released from the microbubbles enter the cells through these perforations, explaining their rapid internalization in Figure 9. Since the lifetime of the cell membrane perforations is very short, ${ }^{[15]}$ it is important that the lipoplexes are closely located to the cell membrane perforations, which is indeed the case when they are attached to the microbubbles. This phenomenon most likely also explains why the physical mixing of the PEGylated lipoplexes with the microbubbles does not dramatically enhance gene transfer (grey bars, part B), since most of the PEGylated lipoplexes are not located close to the microbubbles. As indicated by the arrow in Figure 8, the transfection efficiency of the lipoplex-loaded microbubbles increases as a function of the amount of DSPE-PEG-biotin in the lipoplexes. This can be pacity than "free" PEGylated lipoplexes and naked pDNA used in combination with microbubbles and ultrasound. Interestingly, the lipoplex-loaded microbubbles only transfect cells when exposed to ultrasound, which is promising for space- and time-controlled gene transfer. ${ }^{[30,31]}$ Thus far, the lack of gene transfer has impeded the clinical evaluation of PEGylated lipoplexes. To the best of our knowledge, we have demonstrated for the first time that gene transfer by lipoplexes containing $>5 \mathrm{~mol} \%$ PEG-lipid can be strongly improved by attaching them to microbubbles and exposing them to ultrasound energy. The microbubbles presented in this study are also expected to be suitable for systemic applications. Indeed, microbubbles are already routinely injected in the clinic to enhance the ultrasound-mediated visualization of blood vessels, whereas highly PEGylated lipoplexes are known not to aggregate in blood and have been shown to be harmless. ${ }^{[4,5]}$ Moreover, this novel Trojan-horse-like concept can be used to achieve the ultrasound-controlled delivery of drug-loaded liposomes by simply attaching the drug-loaded liposomes or nanoparticles to the microbubbles. In this way, a more targeted delivery of drugloaded nanoparticles can be achieved, resulting in an increase of the therapeutic index of the drugs. 


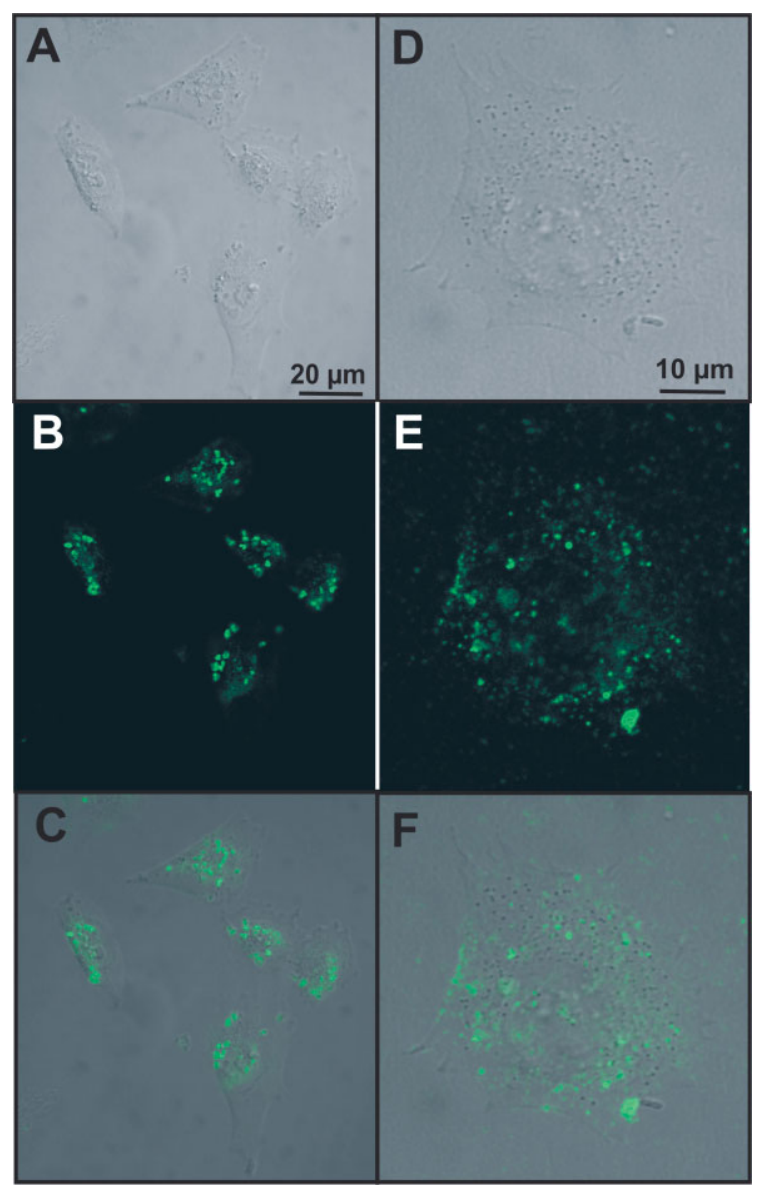

Figure 9. Cellular uptake of green-labeled 15 mol\% PEGylated lipoplexes in BLM cells immediately after exposure of the cells to lipoplex-loaded microbubbles and ultrasound. A-D) Transmission images and B-E) CLSM images of green-labeled lipoplexes; $\mathrm{C}-\mathrm{F}$ ) overlays of the transmission and the green fluorescent confocal images. D-F) High-magnification images of a single cell.

\section{Experimental}

Preparation and Characterization of Lipid Microbubbles Containing DSPE-PEG-Biotin: Liposomes containing DPPC/DSPE-PEG-biotin in a 95:5 molar ratio were prepared as previously described [32]. Briefly, the lipids dissolved in chloroform were placed in a round-bottomed flask and the solvent was removed by evaporation followed by flushing with nitrogen. The obtained lipid film was subsequently hydrated in a 4-(2-hydroxyethyl)piperazine-1-ethanesulfonic acid (HEPES) buffer solution (20 mм HEPES, pH 7.4) at a final lipid concentration of $5 \mathrm{mg} \mathrm{mL}^{-1}$; the film was incubated overnight in this solution at $4{ }^{\circ} \mathrm{C}$ to allow the formation of liposomes. The resulting liposomes were first extruded through a polycarbonate membrane (pore size of $0.2 \mu \mathrm{m}$ ) using a mini-extruder (Avanti Polar Lipids, Alabaster, AL, USA). Subsequently, the extruded liposomes were sonicated with a $20 \mathrm{kHz}$ probe (Branson 250 sonicator, Branson Ultrasonics, Danbury, $\mathrm{CT}$, USA $)$ in the presence of perfluorobutane gas $\left(\mathrm{C}_{4} \mathrm{~F}_{10}\right.$, molecular weight (MW) $238 \mathrm{~g} \mathrm{~mol}^{-1}$, F2 Chemicals, Preston, Lancashire, UK). After sonication, the microbubbles were washed (to remove excess lipids) with $3 \mathrm{~mL}$ fresh HEPES buffer, and finally resuspended in $5 \mathrm{~mL}$ of a fresh HEPES buffer solution. To allow the attachment of biotinylated lipoplexes, the biotinylated microbubbles were incubated with $500 \mu \mathrm{L}$ avidin $\left(10 \mathrm{mg} \mathrm{mL}^{-1}\right)$ for $10 \mathrm{~min}$ at room temperature. Subsequently, the microbubbles were centrifuged and washed again with
$3 \mathrm{~mL}$ fresh HEPES buffer. Finally, the microbubbles were resuspended in a $5 \mathrm{~mL}$ HEPES buffer solution. The concentration of the avidinylated microbubbles in the dispersions was determined with the aid of a Burker chamber and a light microscope, and was found to be $4 \times 10^{8}$ microbubbles $\mathrm{mL}^{-1}$. The size distribution of the microbubbles was determined within $10 \mathrm{~min}$ of preparation by laser diffraction (Mastersizer S, Malvern, Worcestershire, UK). To measure the size distribution of the lipoplex-loaded microbubbles, $130 \mu \mathrm{L}$ of the $15 \mathrm{~mol} \%$ DSPE-PEG-biotin-containing lipoplexes was incubated for $5 \mathrm{~min}$ with $1 \mathrm{~mL}$ of the microbubble suspension and the measurement was performed again. The results are expressed as number percentages normalized to the most abundant fraction of microbubbles. All these experiments were performed using microbubbles dispersed in HEPES buffer. For the visualization of avidin on the surface of the biotinylated microbubbles, we incubated the microbubbles with $50 \mu \mathrm{L}$ of Cy5-labeled streptavidin $\left(1 \mathrm{mg} \mathrm{mL}^{-1}\right)$.

Preparation and Characterization of PEGylated Cationic Liposomes and Lipoplexes: The phospholipids, $N$-(1-(2,3-dioleoyloxy)propyl)$N, N, N$-trimethylammonium chloride (DOTAP), dioleoyl phosphatidyl ethanolamine (DOPE), DSPE-PEG, DSPE-PEG-biotin, and cholesteryl 4,4-difluoro-5,7-dimethyl-4-bora-3a,4a-diaza-s-indacene-3-dodecanoate (cholesteryl Bodipy FLC12) were purchased from Avanti Polar Lipids. Cationic liposomes containing DOTAP/DOPE in a 1:1 molar ratio with $0.1 \mathrm{~mol} \%$ cholesteryl Bodipy FLC12 and 0 to $15 \mathrm{~mol} \%$ DSPE-PEG or DSPE-PEG-biotin were prepared as described above. For the preparation of lipoplexes, we used pDNA (pGL3, Promega, Leiden, The Netherlands) containing the luciferase gene from Photinus pyralis as the reporter. The pDNA was amplified in Escherichia co$l i$ and purified as described elsewhere [32]. The pDNA concentration was set at $1.0 \mathrm{mg} \mathrm{mL}^{-1}$ in HEPES buffer taking into account that the absorption of a $50 \mu \mathrm{g} \mathrm{mL}^{-1}$ DNA solution at $260 \mathrm{~nm}$ equals 1 . The pDNA was of high purity as evidenced by the ratios of the optical absorptions at 260 and $280 \mathrm{~nm}$ varying from 1.8 to 2.0. Lipoplexes (with different percentages of DSPE-PEG or DSPE-PEG-biotin) were prepared with a charge ratio of 4 . The charge ratio is defined as the ratio of the number of the positive charges (originating from DOTAP) to the number of the negative charges (originating from the pDNA). pDNA was first diluted in HEPES buffer to a concentration of $0.41 \mathrm{mg} \mathrm{mL}^{-1}$. Subsequently, the diluted pDNA was added to an equal volume of cationic liposomes (5 mM DOTAP), resulting in a final +/charge ratio of 4 . Immediately after the addition of pDNA to the cationic liposomes, HEPES buffer was added until the final concentration of pDNA in the system was $0.126 \mathrm{mg} \mathrm{mL}^{-1}$. This mixture was then vortexed and incubated at room temperature for $30 \mathrm{~min}$. To obtain greenlabeled lipoplexes, liposomes prepared with $0.5 \mathrm{~mol} \%$ of cholesteryl Bodipy FLC12 were used. The average hydrodynamic diameter of the PEGylated lipoplexes was determined by DLS (Autosizer 4700, Malvern). The data were analyzed using the automatic data analysis mode, i.e., a monomodal fit was used when the polydispersity (PD) was $<0.05$ and a continuous fit was used when the PD was $>0.05$. The zeta potential $(\zeta)$ was determined using electrophoretic mobility measurements (Malvern Zetasizer Nano ZS, Malvern). For these experiments, the lipoplexes were dispersed in HEPES buffer. The size and zeta potential of lipoplexes released from the lipoplex-loaded microbubbles after exposure to ultrasound $(1 \mathrm{MHz}, 10 \%$ duty cycle) for $10 \mathrm{~s}$ were determined in a similar way. Gel electrophoresis experiments were performed following previously described protocols [32] to determine the presence of free pDNA in the lipoplexes before binding to the microbubbles and after ultrasound-assisted release from the microbubbles.

Attachment of Biotinylated PEG-Lipoplexes to Avidinylated Microbubbles: $130 \mu \mathrm{L}$ of a solution of biotinylated PEG-lipoplexes was mixed with a $1 \mathrm{~mL}$ solution of avidinylated mirobubbles and incubated at room temperature for $5 \mathrm{~min}$. The attachment of fluorescent-labeled lipoplexes to the microbubbles was visualized by CLSM using a Nikon EZC1-si microscope (Nikon, Brussels, Belgium) equipped with a $40 \times$ objective. The $491 \mathrm{~nm}$ line of this microscope was used to excite the Bodipy label.

Transfection Experiments: BLM cells (melanoma cells) [33] were cultured in Dulbecco's modified Eagle's medium (DMEM) with the growth factor F12 and phenol red containing 2 mM glutamine, $10 \%$ 
heat-deactivated fetal bovine serum (FBS), $1 \%$ penicillin-streptomycin (Gibco, Merelbeke, Belgium), and HEPES buffer (100 mm, $\mathrm{pH}$ 7.4). The cells were grown to $90 \%$ confluency in Opticell units (Biocrystal, Westerville, OH, USA) inside a humidified incubator at $37^{\circ} \mathrm{C}$ under $5 \% \mathrm{CO}_{2}$. Subsequently, the cells were washed with $10 \mathrm{~mL}$ of phosphate buffered saline (PBS, Gibco) and the transfection medium was added. The transfection media were prepared by first mixing $130 \mu \mathrm{L}$ PEGylated lipoplexes with $1 \mathrm{~mL}$ of the microbubble suspension (containing $4 \times 10^{8}$ microbubbles). After incubation for $5 \mathrm{~min}$ at room temperature, optimem (Gibco) was added to a final volume of $10 \mathrm{~mL}$. The transfection medium was prepared in a similar way for transfection experiments with naked pDNA, except that the $130 \mu \mathrm{L}$ solution of the PEGylated lipoplexes was replaced by an equal volume of HEPES buffer containing $16.5 \mu \mathrm{g}$ pDNA, the same amount as present in the lipoplexes. $10 \mathrm{~mL}$ of the transfection medium was added to the Opticell units (surface area of $50 \mathrm{~cm}^{2}$ ). Subsequently, the cells were placed in a water bath at $37^{\circ} \mathrm{C}$ with an absorbing rubber substrate at the bottom and immediately subjected to ultrasound irradiation. The ultrasound irradiation was performed for $10 \mathrm{~s}$ with a Sonitron 2000 instrument (RichMar, Inola, OK, USA) equipped with a $22 \mathrm{~mm}$ probe. In all the ultrasound-assisted experiments, the following ultrasound settings were used: $1 \mathrm{MHz}, 10 \%$ duty cycle, and an ultrasound intensity of $2 \mathrm{~W} \mathrm{~cm}^{-2}$. The areas treated with ultrasound were marked and after irradiation the Opticells were incubated for an additional $2 \mathrm{~h}$ at $37^{\circ} \mathrm{C}$. At the end of this incubation period, the transfection medium was removed and the cells were washed two times with PBS, before adding fresh culture medium. Each transfection experiment was performed three times. Luciferase expression by the cells was analyzed $24 \mathrm{~h}$ after transfection. The culture medium was removed and the cells were washed with PBS. The areas exposed to ultrasound ( $20 \mathrm{~mm}$ diameter) were cut from the Opticell membrane and placed in a 24 well plate. A $80 \mu \mathrm{L}$ solution of cell culture lyse reagent (CCLR, Promega, Leiden, The Netherlands) buffer was added to each well and incubated at room temperature for at least 20 min to allow cell lysis. $20 \mu \mathrm{L}$ of the cell lysate was transferred to a 96 well plate and the luciferase activity was measured using a Glomax 96 Microplate Luminometer (Promega), as described previously in the literature [34]. An aliquot $(20 \mu \mathrm{L})$ of each cell lysate was also analyzed for protein concentration using the bichinconinic acid (BCA) protein assay (Pierce, Rockford, IL, USA). The transfection results are expressed as relative light units (RLU) per milligram of protein.

Cellular Uptake of Green-Labeled $15 \mathrm{~mol} \%$ DSPE-PEG-BiotinContaining Lipoplexes: Bodipy-labeled lipoplexes containing $15 \mathrm{~mol} \%$ DSPE-PEG-biotin were prepared and attached to the microbubbles as described above. BLM cells present in Opticell units were exposed to the lipoplex-loaded microbubbles and ultrasound using the same conditions as in the transfection experiments. The areas treated with ultrasound were immediately visualized by CLSM using a Nikon EZC1-si microscope equipped with a $60 \times$ objective. The $491 \mathrm{~nm}$ line of this microscope was used to excite the Bodipy label in the lipoplexes.

Statistical Analysis: All the data in this report are expressed as mean \pm standard deviation (SD). For the transfection results, the student's t-test was used to determine whether the data groups differed significantly from each other. A $p$-value lower than 0.05 was considered statistically significant.

Received: January 24, 2007 Revised: May 16, 2007 Published online: July 4, 2007

[1] S. D. Patil, D. G. Rhodes, D. J. Burgess, AAPS J. 2005, 7, E61.

[2] E. Marshall, Science 1999, 286, 2244.
[3] H. Eliyahu, N. Servel, A. J. Domb, Y. Barenholz, Gene Ther. 2002, 9 , 850.

[4] F. Sakurai, T. Nishioka, F. Yamashita, Y. Takakura, M. Hashida, Eur J. Pharm. Biopharm. 2001, 52, 165.

[5] S. M. Moghimi, J. Szebeni, Prog. Lipid Res. 2003, 42, 463.

[6] L. Wasungu, D. Hoekstra, J. Controlled Release 2006, 116, 255.

[7] I. S. Zuhorn, J. B. Engberts, D. Hoekstra, Eur. Biophys. J. 2007, 26, 349.

[8] R. Bekeredjian, S. Y. Chen, P. A. Frenkel, P. A. Grayburn, R. V. Shohet, Circulation 2003, 108, 1022

[9] M. Duvshani-Eshet, D. Adam, M. Machluf, J. Controlled Release 2006, 112,156

[10] M. Kinoshita, K. Hynynen, Biochem. Biophys. Res. Commun. 2005 , 335, 393.

[11] Y. Manome, N. Nakayama, K. Nakayama, H. Furuhata, Ultrasound Med. Biol. 2005, 31, 693.

[12] C. M. Newman, A. Lawrie, A. F. Brisken, D. C. Cumberland, Echocardiography 2001, 18, 339.

[13] S. V. Pislaru, C. Pislaru, R. R. Kinnick, R. Singh, R. Gulati, J. F. Greenleaf, R. D. Simari, Eur. Heart J. 2003, 24, 1690.

[14] M. Vannan, T. McCreery, P. Li, Z. G. Han, E. Unger, B. Kuersten, E. Nabel, S. Rajagopalan, J. Am. Soc. Echocardiogr. 2002, 15, 214.

[15] S. Mehier-Humbert, T. Bettinger, F. Yan, R. H. Guy, J. Controlled Release 2005, 104, 213.

[16] R. K. Schlicher, H. Radhakrishna, T. P. Tolentino, R. P. Apkarian, V. Zarnitsyn, M. R. Prausnitz, Ultrasound Med. Biol. 2006, 32, 915

[17] A. Van Wamel, A. Bouakaz, M. Versluis, N. De Jong, Ultrasound Med. Biol. 2004, 30, 1255 .

[18] E. C. Unger, T. Porter, W. Culp, R. Labell, T. Matsunaga, R. Zutshi, Adv. Drug Delivery Rev. 2004, 56, 1291.

[19] A. F. H. Lum, M. A. Borden, P. A. Dayton, D. E. Kruse, S. I. Simon, K. W. Ferrara, J. Controlled Release 2006, 111, 128

[20] M. C. Deshpande, M. C. Davies, M. C. Garnett, P. M. Williams, D. Armitage, L. Bailey, M. Vamvakaki, S. P. Armes, S. Stolnik, J. Controlled Release 2004, 97, 143.

[21] S. Audouy, D. Hoekstra, Mol. Membr. Biol. 2001, 18, 129

[22] O. Meyer, D. Kirpotin, K. L. Hong, B. Sternberg, J. W. Park, M. C. Woodle, D. Papahadjopoulos, J. Biol. Chem. 1998, 273, 15621.

[23] S. Mishra, P. Webster, M. E. Davis, Eur. J. Cell Biol. 2004, 83, 97.

[24] F. X. Shi, L. Wasungu, A. Nomden, M. C. A. Stuart, E. Polushkin, J. B. F. N. Engberts, D. Hoekstra, Biochem. J. 2002, 366, 333.

[25] L. Y. Song, Q. F. Ahkong, Q. Rong, Z. Wang, S. Ansell, M. J. Hope, B. Mui, Biochim. Biophys. Acta-Biomembr. 2002, 1558, 1

[26] N. W. Andrews, Trends Cell Biol. 2000, 10, 316.

[27] K. Tachibana, T. Uchida, K. Ogawa, N. Yamashita, K. Tamura, Lancet 1999, 353, 1409.

[28] A. van Wamel, K. Kooiman, M. Harteveld, M. Emmer, F. J. ten Cate, M. Versluis, N. de Jong, J. Controlled Release 2006, 112, 149.

[29] I. Lentacker, B. G. De Geest, R. E. Vandenbroucke, L. Peeters, J. Demeester, S. C. De Smedt, N. N. Sanders, Langmuir 2006, 22, 7273.

[30] A. L. Klibanov, Investigative Radiol. 2006, 41, 354.

[31] Y. Y. Liu, H. Miyoshi, M. Nakamura, J. Controlled Release 2006, 114, 89.

[32] N. N. Sanders, E. Van Rompaey, S. C. De Smedt, J. Demeester, Am. J. Respir. Crit. Care Med. 2001, 164, 486.

[33] P. H. A. Quax, G. N. P. Vanmuijen, E. J. D. Weeningverhoeff, L. R. Lund, K. Dano, D. J. Ruiter, J. H. Verheijen, J. Cell Biol. 1991, 115, 191.

[34] K. von Gersdorff, N. N. Sanders, R. Vandenbroucke, S. C. De Smedt, E. Wagner, M. Ogris, Mol. Ther. 2006, 14, 745. 